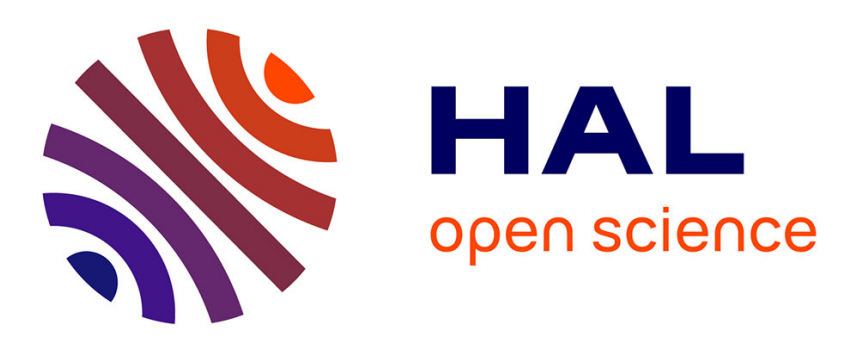

\title{
A Nonlinear PID Stabilizer With Spherical Projection for Humanoids: From Concept to Real-time Experiments
}

David Galdeano, Ahmed Chemori, Sébastien Krut, Philippe Fraisse

\section{- To cite this version:}

David Galdeano, Ahmed Chemori, Sébastien Krut, Philippe Fraisse. A Nonlinear PID Stabilizer With Spherical Projection for Humanoids: From Concept to Real-time Experiments. Humanoids, Nov 2014, Madrid, Spain. pp.693-698, 10.1109/HUMANOIDS.2014.7041438 . lirmm-01089549

\section{HAL Id: lirmm-01089549 \\ https://hal-lirmm.ccsd.cnrs.fr/lirmm-01089549}

Submitted on 2 Dec 2014

HAL is a multi-disciplinary open access archive for the deposit and dissemination of scientific research documents, whether they are published or not. The documents may come from teaching and research institutions in France or abroad, or from public or private research centers.
L'archive ouverte pluridisciplinaire HAL, est destinée au dépôt et à la diffusion de documents scientifiques de niveau recherche, publiés ou non, émanant des établissements d'enseignement et de recherche français ou étrangers, des laboratoires publics ou privés. 


\title{
A Nonlinear PID Stabilizer With Spherical Projection for Humanoids: From Concept to Real-time Experiments
}

\author{
David Galdeano ${ }^{1}$, Ahmed Chemori ${ }^{1}$, Sébastien Krut $^{1}$ and Philippe Fraisse ${ }^{1}$
}

\begin{abstract}
This paper deals with a stabilizer for a hybrid kinematic/dynamic control scheme for humanoid robots. The proposed solution is based on a nonlinear PID regulation of the ZMP, coupled with a spherical projection in the CoM's control space. The result of such a stabilizer is a dynamically stable motion, even with large variations in the inclination of the ground. The effectiveness of this new stabilizer has been demonstrated through real-time experiments on the humanoid robot HOAP-3. The produced motion is smooth and dynamically stable.
\end{abstract}

\section{INTRODUCTION}

The goal of developing humanoid robots is to make them working in dull, dirty and dangerous environments where human workers could be in danger. However, up to now, most of humanoid robots are still confined to research laboratories, since they are not reliable enough in real operating conditions. One of the main features that should be improved to produce reliable humanoid robots is the stability of the motions. Their fall can be dangerous for humans standing in a close proximity of the robots. The robot ability to move in its environment, even if the environment is degraded, is needed to bring robots to consumers application.

Even with an optimal joint trajectory design, the stability of the motion could not be guaranteed if a perturbation occurs. In order to produce stable motions, the robot movement should be adapted online to the external environment using the sensors feedback information. This reactive adaptation allows the robot to evolve in a real world environment. The stability indicators are mathematical tools to quantify the degree of stability stability. Mainly two indicators of stability can be used: the Center of Mass (CoM) and the Zero Moment Point (ZMP).

The COM is the mean location of all masses of the robot's links. This indicator is often used for static stability [1]. The ZMP [2] is the point of junction between the resultant vertical reaction force and the ground. It is the most used indicator for dynamic stability [3], [4]. In the literature, various methods have been proposed to improve the stability of the robot motions based on the ZMP stability indicator.

One of the most used methods is called the Inverted Pendulum Model (IPM) [5], [6] that considers the robot as a single pointwise mass and massless legs. This method simplifies the dynamics of the robot by using the relationship between the CoM and the ZMP. The robot's dynamics is approximated by the one of an inverted pendulum with

\footnotetext{
${ }^{1}$ LIRMM, Université Montpellier 2, LIRMM - UMR 5506 - CC 477, 161 rue Ada, 34095 Montpellier Cedex 5, France galdeano (chemori, krut, fraisse) @lirmm.fr
}

a pointwise mass linked through a telescopic leg to a spherical ground/leg joint. Since then, different extensions have been proposed such as the Linear Inverted Pendulum Model (LIPM) [7], [8]. The preview control of the ZMP [9] has improved the LIPM by reducing the error induced by the simplification of the dynamics of the humanoid robot. The previous three methods are very efficient to perform walking motions, but the model equations are based on the assumption that the ground is flat, therefore the range of admissible motions is limited. The ZMP preview control can be extended by using a model predictive control (MPC)to be more robust against unexpected external disturbances [10].

A control law can be design to allows a compliant interaction with external forces. This technique have been implemented on several robots to allows the generation of stables motions by absorbing the external perturbations [11], [12]. However, this technique requires a torque level control [13] to be efficient which is not possible on all humanoids robots.

Some other proposed techniques are based on a sensory feedback system [14], [15]. A stabilizer can be used to control the torque of the ankle joint [16], [17], [18], the angular momentum of the hip [19], [20], the trajectory of the CoM [21], [22] or the decision to take a step [23], [24] in order to ensure the motion stability. The above approaches are very reactive and can adapts to large disturbances. However, they are difficult to be combined with frameworks using the rest of the body of the robot in other useful tasks.

In this paper, a nonlinear ZMP based stabilizer is proposed. This stabilizer is sensory feedback based and has been developed within the framework of an hybrid dynamic/kinematic whole-body control strategy. The whole control architecture with a simpler stabilizer has been proposed in a previous works of the authors [25]. The basic idea of this stabilizer is to use a nonlinear PID controller to regulate the ZMP error and to project this regulation in a CoM tracking objective using a spherical projection. The nonlinear PID controller allows a fast tracking with a favorable damping, improving the stability of the controlled robot. The spherical projection of the ZMP error compensation allows to adapt the robot posture to large variations in the inclination of the ground.

The proposed stabilizer allows to increase the rejection of external disturbances applied to the humanoid robot.

In the literature, most of the proposed stabilizers relies on precomputed trajectories for the desired ZMP trajectory. With the proposed stabilizer, the regulation of the center of pressure $(\mathrm{CoP})$ is used to produce dynamically stables 
motions. The stabilizer has been implemented on HOAP3 , a small robot with position control, noisy sensors data and low computational power. The control scheme can be easily deployed on other robots if they are equipped with joint encoders and CoP sensors on both feet.

This paper is organized as follows: in next section, our humanoid robot demonstrator HOAP-3 is introduced. Section II is devoted to the proposed nonlinear ZMP based stabilizer. The ZMP regulation is mapped in the CoM's control space using a spherical projection. In this section, its basic principle is presented and discussed. Real-time experimental results are introduced in section III, with a presentation and discussion of the obtained results. The paper ends with some concluding remarks and an overview on future work.

\section{MAIN CONTRIBUTION: A ZMP ERROR PROJECTION BASED NONLINEAR STABILIZER}

\section{A. General overview of the control scheme}

In a previous work of the authors, a hybrid kinematic/dynamic whole-body control framework has been proposed [25]. This framework has been experimentally validated for squat-like motions. The framework consist in a kinematic control including four objectives, namely (i) the robot's feet relative-pose, (ii) the CoM, (iii) the body orientation and (iv) joints' limits avoidance. The kinematic control is based on the task formalism [26], [27].

A ZMP based dynamic feedback has been considered to produce a dynamically stable motions. This stabilizer uses the ZMP error to modify the CoM trajectory. In this work, we aim at improving this stabilizer through two main contributions:

- A nonlinear feedback control of the ZMP error to improve the rapidity and stability of the stabilizer,

- a sphere projection of this regulation to produce humanlike motion even with large variations in the inclination of the ground.

\section{B. Nonlinear ZMP regulation controller}

Nonlinear PD (NPD) controllers have been proposed in robotics field as an improvement of the classic linear PD controllers [28], [29], [30], [31]. The nonlinear ZMP regulation control is used to produce a reactive control with a better damping [31]. This allows a faster response without introducing instabilities.

Contrary to classical linear PD controller, the NPD controller uses time-varying gains depending on the tracking errors instead of fixed gains. The general expression of the NPD can be expressed as:

$$
u(t)=k_{p}(\cdot) e(t)+k_{d}(\cdot) \dot{e}(t)
$$

where $k_{p}(\cdot)$ and $k_{d}(\cdot)$ are the time-varying proportional and derivative gains, $e(t)$ is the system error and $\dot{e}(t)$ its first time derivative (velocity error).

The time-varying proportional and derivative gains may depend on the system state, its inputs or other variables. In our case, we consider a proportional gain depending on the position error and a derivative gain depending on the velocity error.

The nonlinear proportional gain, illustrated in Fig. 1, is expressed by:

$$
k_{p}(e)= \begin{cases}k_{p_{0}}|e|^{\alpha_{1}-1}, & |e|>\delta_{1}, \\ k_{p_{0}} \delta_{1}^{\alpha_{1}-1}, & |e| \leqslant \delta_{1} .\end{cases}
$$

where $k_{p_{0}}$ is the maximum proportional gain, $\alpha_{1}$ is the nonlinearity tuning parameter and $\delta_{1}$ its threshold of activation.

The nonlinear derivative gain, also illustrated in Fig. 1, is given by:

$$
k_{d}(\dot{e})= \begin{cases}k_{d_{0}}|\dot{e}|^{\alpha_{2}-1}, & |\dot{e}|>\delta_{2}, \\ k_{d_{0}} \delta_{2}^{\alpha_{2}-1}, & |\dot{e}| \leqslant \delta_{2} .\end{cases}
$$

where $k_{d_{0}}$ is the maximum derivative gain, $\alpha_{2}$ is the nonlinearity tuning parameter and $\delta_{2}$ its threshold of activation.

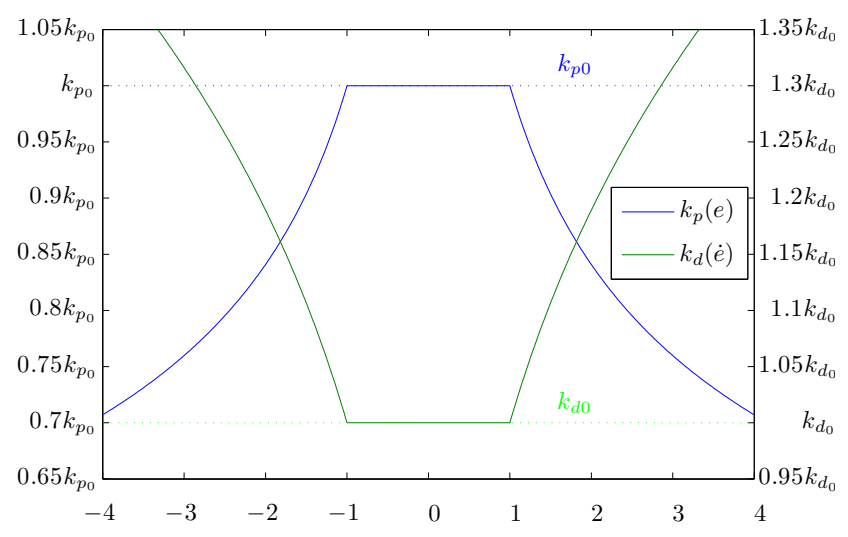

Fig. 1: Typical evolution of the nonlinear proportional $k_{p}$ and derivative $k_{d}$ gain versus position error $e$ and velocity error $\dot{e}$ with $\alpha_{1}=0.75, \delta_{1}=1, \alpha_{2}=1.25$ and $\delta_{2}=1$.

The parameter tuning for $\alpha_{1}, \delta_{1}, \alpha_{2}, \delta_{2}$ is explain in [31]. We consider $\alpha_{1} \in[0.5,1.0]$ and $\alpha_{2} \in[1.0,1.5]$ to produce a fast trajectory tracking with improved disturbance rejections.

A NPD controller is not designed to compensate static errors since it does not contain an integral action. Consequently an integral action should be considered to compensate the ZMP static errors. We propose to define the ZMP error compensation as an offset to modify the CoM tracking in order to improve the robot's dynamic stability. The ZMP error compensation can then be defined as:

$$
\mathbf{o} \mathbf{Z M P}=k_{p}\left(\varepsilon_{\mathbf{Z M P}}\right) \varepsilon_{\mathbf{Z M P}}+k_{d}\left(\frac{d \varepsilon_{\mathbf{Z M P}}}{d t}\right) \frac{d \varepsilon_{\mathbf{Z M P}}}{d t}+k_{i} \int \varepsilon_{\mathbf{Z M P}}
$$

where o ZMP $\in \mathbb{R}^{2 \times 1}$ is the ZMP error compensation, $k_{i}$ is the integral gain and $\varepsilon_{\mathbf{Z M P}} \in \mathbb{R}^{2 \times 1}$ the ZMP tracking error.

The ZMP tracking error is computed from the center of pressure (CoP) measurement as presented in [25]. One of the main advantage of this approach is that the ZMP tracking error, $\varepsilon_{Z M P}$ is a weighted distribution of ZMP errors on both feet. The ZMP error compensation is not computed using a 
ZMP reference trajectory in global coordinates but rather a local coordinates CoP reference trajectory since the desired $\mathrm{CoP}$ is at the center of each feet.

\section{Spherical projection of the ZMP regulation}

In the control scheme proposed in [25], the ZMP error compensation was directly added to the CoM objective as a planar projection. Since the CoM trajectory was modified in the transverse plane, this concept was limited to small ZMP errors and flat ground. However, for the case of large ZMP errors or the case of an inclined ground, the ZMP error compensation should be carefully managed to avoid structural singularities produced by stretching the desired CoM out of reach as illustrated in 2(a).

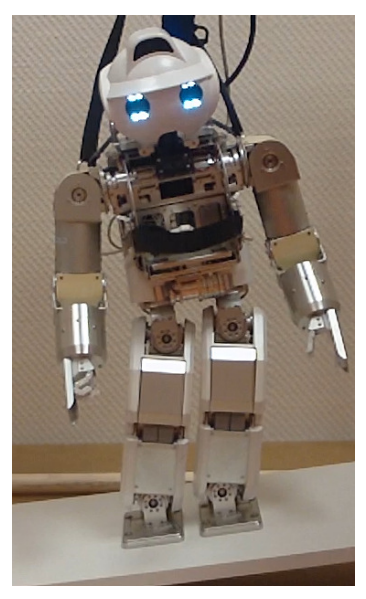

(a) Planar projection.

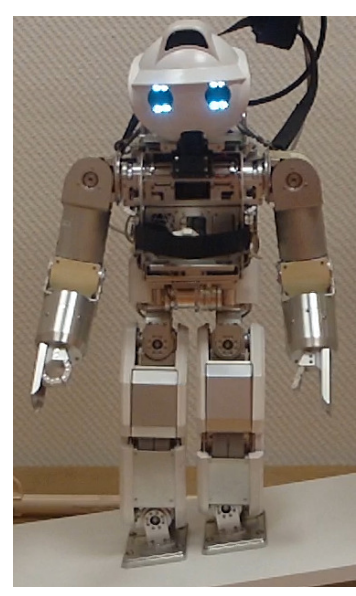

(b) Spherical projection.
Fig. 2: Illustration of the real-time adaptation against ground's inclination with two different projections.

The proposed solution is then to project the ZMP error compensation on a virtual sphere (as illustrated in Fig. 2(b)). This sphere is defined by its center positioned on the ground, at the ZMP position, and its radius is equal to the initial CoM height as illustrated on Fig. 3.

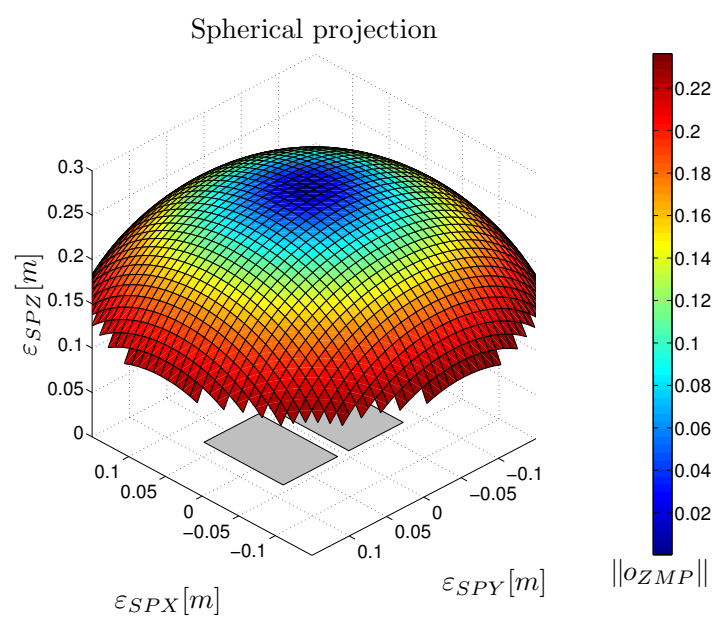

Fig. 3: Illustration of the sphere projection space w.r.t. the norm of $o_{Z M P}(\beta=1$ and $\gamma=1)$. Footprints are displayed in gray.
The spherical projection equation of the ZMP error compensation $\varepsilon_{\mathbf{S P}}=\left[\begin{array}{lll}\varepsilon_{S P X} & \varepsilon_{S P Y} & \varepsilon_{S P Z}\end{array}\right]^{T}$, with $\mathbf{o}_{\mathbf{Z M P}}=$ $\left[\begin{array}{ll}o_{Z M P X} & o_{Z M P Y}\end{array}\right]^{T}$ from eq. (4), can be expressed as:

$$
\begin{aligned}
& \varepsilon_{S P X}=h_{C o M} \sin \left(\frac{\beta \cdot o_{Z M P X}}{h_{C o M}}\right), \\
& \varepsilon_{S P Y}=h_{C o M} \sin \left(\frac{\gamma \cdot o_{Z M P Y}}{h_{C o M}}\right), \\
& \varepsilon_{S P Z}=\sqrt{h_{C o M}{ }^{2}-o_{Z M P X}{ }^{2}-o_{Z M P Y}{ }^{2}} .
\end{aligned}
$$

where $h_{C o M}$ denotes the initial CoM height, $\beta$ and $\gamma$ are spherical projection adjustable parameters that allows to tune the shape of the spherical projection to stay inside the kinematic workspace of the legs.

The projection of the ZMP error compensation is then added as an offset to the CoM tracking as follows:

$$
\varepsilon_{\mathbf{C Z}}=\varepsilon_{\mathbf{C o M}}+\varepsilon_{\mathbf{S P}}
$$

where $\varepsilon_{\mathbf{C Z}} \in \mathbb{R}^{2 \times 1}$ is the tracked stability objective containing the CoM tracking and the ZMP regulation; and $\varepsilon_{\mathbf{C o M}} \in \mathbb{R}^{2 \times 1}$ is the CoM tracking error [25].

The Fig. 4 illustrate the CoM trajectory in the frontal plane if a disturbance is applied on the ZMP on $y$ axis. This projection space has been verified to be inside the kinematic workspace of the legs if $\beta=1$ and $\gamma=1$.

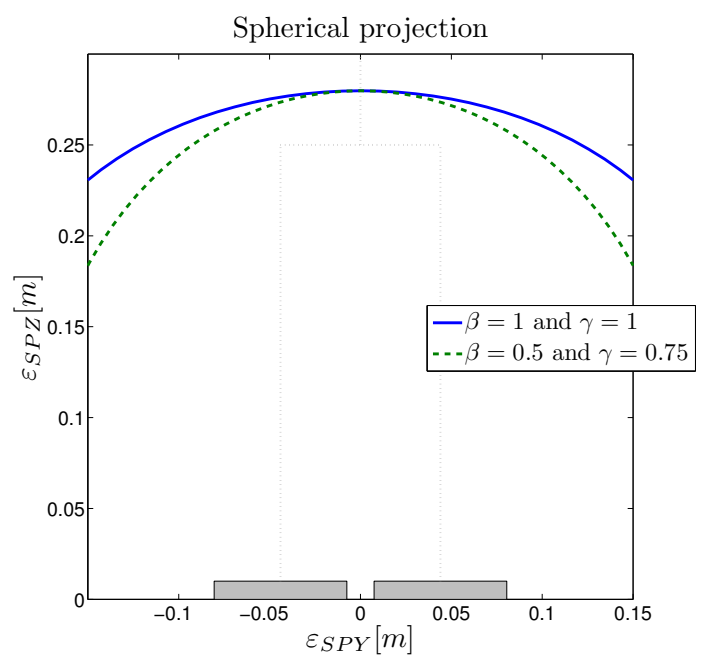

Fig. 4: Frontal view of the sphere projection with $u_{Z M P X}=$ 0 and $u_{Z M P X}$ varying in $\left[-h_{C o M} \frac{\pi}{2} ; h_{C o M} \frac{\pi}{2}\right]$ domain. Robot's structure and footprints are displayed in gray. Spherical projection tuning parameter are set to $\beta=1, \gamma=1$ for the solid line and $\beta=0.5, \gamma=0.75$ for the dashed line.

In order to produce an human-like reaction to slope variation of the ground and to avoid auto-collision of the legs of the robot, the torso orientation must be managed. Since the upper-body orientation is not applied on the CoM tracking but on the torso orientation tracking, this does not modify the stability of the robot. The desired torso orientation becomes:

$$
\begin{aligned}
& \text { Torso_ori }_{d}(x)=\operatorname{atan} 2\left(o_{Z M P Y}, h_{C o M}\right) \\
& \text { Torso_ori }_{d}(y)=\operatorname{atan} 2\left(o_{Z M P X}, h_{C o M}\right) .
\end{aligned}
$$


where Torso_ori $i_{d}(x)$ and Torso_ori $i_{d}(y)$ are the desired torso orientations. The desired torso orientation is managed in a different regulation objective than the stability objective.

This stabilizer improves the stability locally by modifying the reference position of the CoM. The quick reaction of the ZMP-based stabilizer to $\mathrm{CoP}$ errors allows to converge to stable motions if quick and small perturbations occurs. Large and slow disturbances are taken into account using the integral part and the spherical projection to allows a stable posture on inclined ground. It does not guaranties a global stability, which needs the decision of taking a step to keep stability in case of quick and large disturbances.

\section{REAL-TIME EXPERIMENTAL VALIDATION}

The proposed stabilizer presented in section II has been implemented within the control scheme proposed in [25] on the HOAP-3 humanoid robot presented in the sequel. Two experimental scenarios have been performed to show the efficiency of the proposed stabilizer.

In the first scenario, the slope of the ground has been varied to demonstrate the adaptation of the proposed stabilizer to an inclined ground. The second scenario demonstrates a task of dynamic walking on uneven ground with an unexpected variation in the slope of the ground. In the sequel, the experimental setup and the two scenarios will be detailed.

\section{DESCRIPTION OF THE EXPERIMENTAL SETUP}

The proposed control scheme within this work has been be implemented on HOAP-3 humanoid robot, manufactured by Fujitsu Automation. The experimental setup for the following validations consist in a HOAP-3 robot communicating with a host PC as illustrated in Fig. 5.

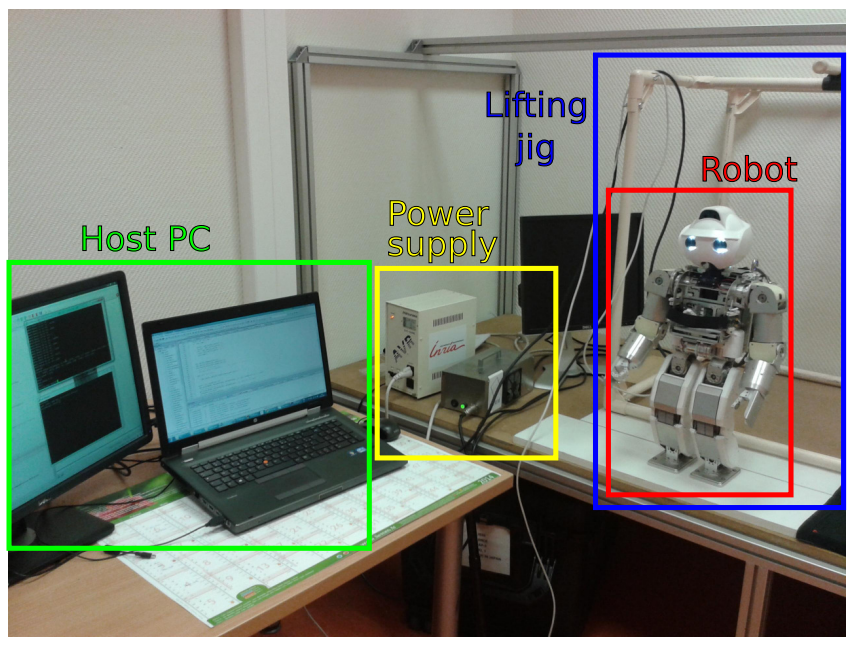

Fig. 5: The experimental setup for the real-time experiments consist in the HOAP-3 humanoid robot linked to a host PC.

The robot weights $8.8 \mathrm{~kg}$ and is $60 \mathrm{~cm}$ tall. Its mechanical structure is composed of 28 degrees of freedom (dof) distributed as follows: 6 dof per leg, one in the waist, 5 per arm, 3 for the neck and one per hand.

The robot is equipped with an incremental encoder per joint and four force sensors per feet wich have been used for this work. Beside, it is equipped with a three-axis acceleration sensor, a three-axis gyro sensor and two CDD cameras which have not been used.

A control PC with RT-Linux real-time operating system allows the control of the robot with a sampling time of 1 msec. This robot is a versatile testbed for whole-body motion control. Our stabilizer algorithm is implemented in a $\mathrm{C}$ language code for real-time experiments.

\section{A. Scenario 1: Online adaptation toward slope variation of the ground}

The objective of this scenario is to demonstrate the adaptation of the proposed stabilizer against slope variation of the ground. The experimental test platform consist of a wooden board (as shown in Fig. 6) which is lifted from one side, therefore creating a rotation around the opposite edge of the board. The spherical projection tuning parameter are $\beta=1$ and $\gamma=1$ for this scenario.

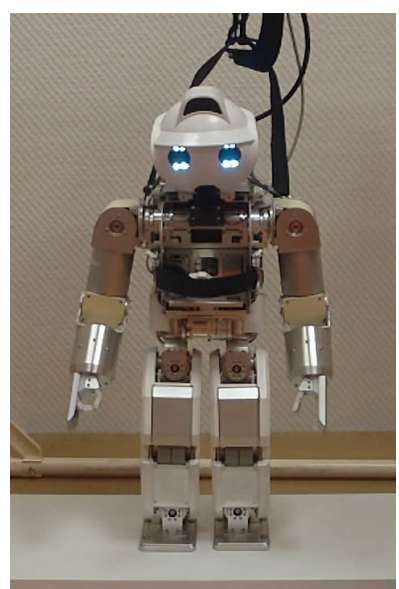

(a) Experimental setup

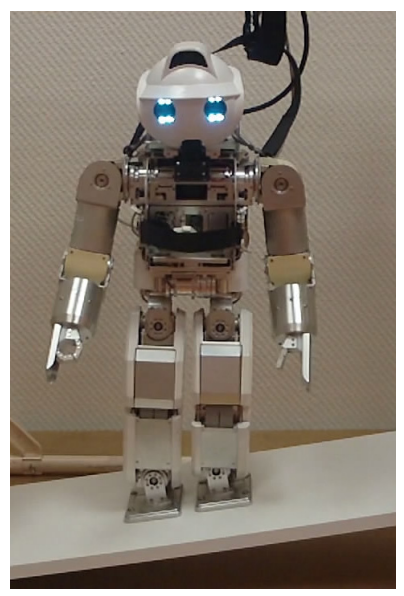

(b) Large ground's inclination
Fig. 6: Illustration of the real-time adaptation against ground's inclination (scenario 1).

The robot adaptation to the ground's inclination variation can be observed on the illustration of Fig. 6 .

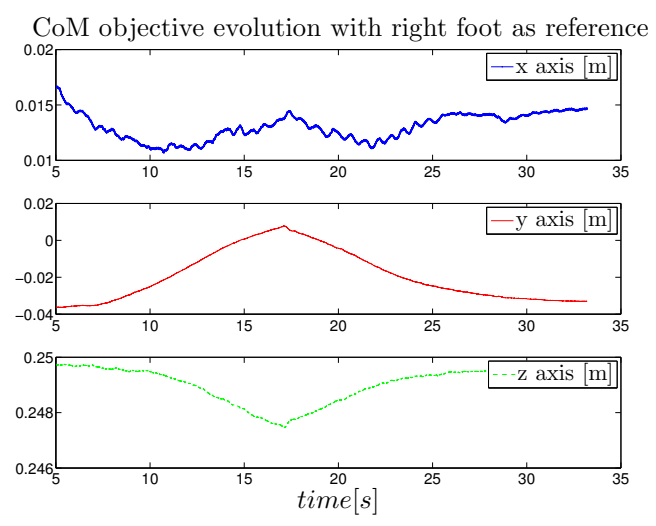

Fig. 7: Trajectories evolution of the CoM versus time for the first scenario.

In Fig. 7, the evolution of the CoM position (Cartesian coordinates) expressed in the right foot's reference coordinate is displayed. Along the $x$ axis, the trajectory is constant since 
no perturbation on this axis has been introduced. Along the $y$ axis, the trajectory of the CoM should be regulated around its desired value (constant). Indeed, the observed variation is due to the adaptation of the stabilizer to the ZMP displacement. The CoM trajectory is modify to keep balance. Along the $z$ axis, the trajectory is also kept constant at the height of the initial CoM position.

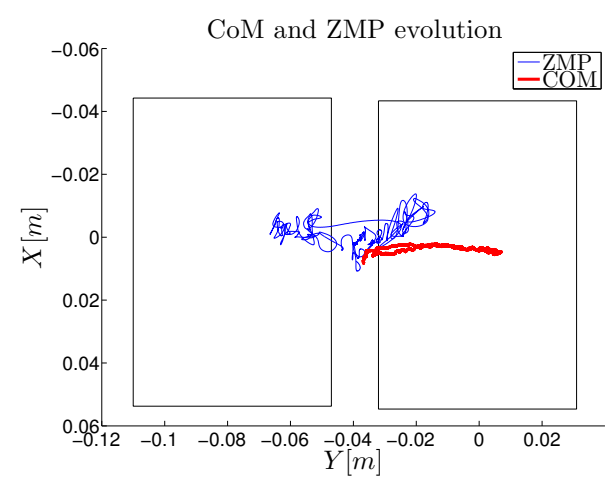

Fig. 8: Evolution of the CoM and ZMP trajectories with respect to footprints (scenario 1).

In Fig. 8, the evolution of the measured ZMP and CoM positions are plotted with respect to the footprints of the robot. The trajectory of the desired CoM is constant. The observed variation on the CoM position is due to the adaptation of the stabilizer to the ZMP displacement to keep balance.

The robot's body adapts to ground's inclination variation. The combination of the CoM position adjustment and the hip rotation allow a smooth motion. The posture of the robot during large ZMP disturbances on Fig. 6(b) is looking natural, the torso is not inclined like the ground.

\section{B. Scenario 2: Walking on an uneven ground}

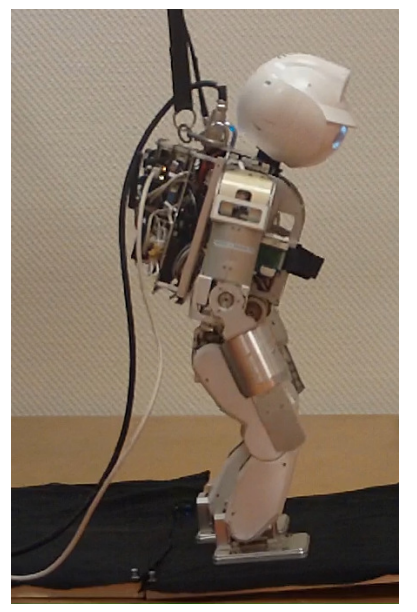

Fig. 9: Illustration of walking on unexpected inclined ground (scenario 2).

The objective of this scenario is to show the robustness of the control scheme against ground's slope variation while walking. The robot is walking on a flat ground followed by a five degrees inclined plane as illustrated in Fig. 9. A simple B-splines based pattern generator under Matlab ${ }^{1}$ software

\footnotetext{
${ }^{1}$ Mat lab is a registered trademark of The Mathworks, Inc.
}

is used to produce the desired CoM position and the feet relative pose trajectories.

This pattern generator was used to produce the feet and the CoM trajectories in operational space. The objectives' trajectories have been designed to produce a walk of six steps. The produced feet and CoM trajectories are then expressed in the right foot reference coordinates in order to be used as reference trajectories. The spherical projection tuning parameter are $\beta=1$ and $\gamma=1$ for this scenario.
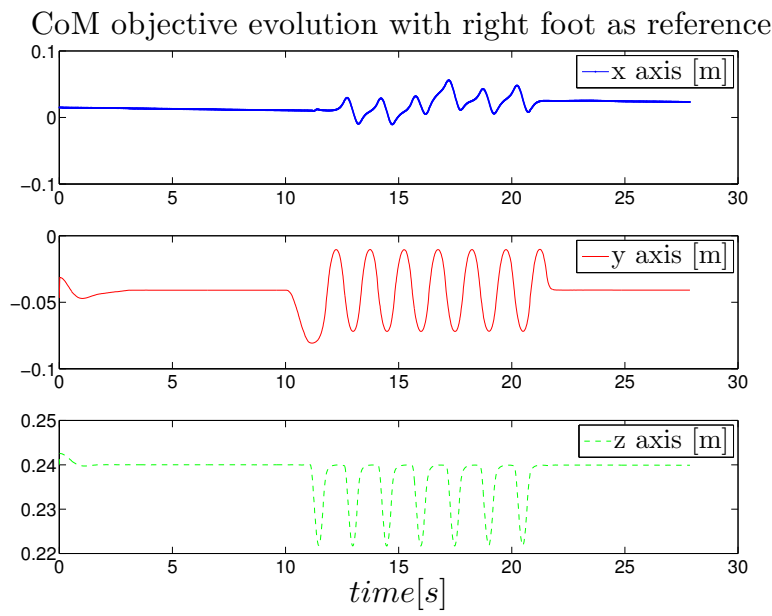

Fig. 10: Trajectories evolution of the CoM versus time for the second scenario.

In Fig. 10, the evolution of the CoM position expressed in the right foot reference coordinates is displayed. On the $x$ axis, the trajectory is similar to the classical trajectory given by an inversed pendulum model, except that the ZMP regulation shifts the CoM forward (increase on $x$ axis) to maintain the stability of the robot when walking on the inclined ground. On the $y$ axis, the trajectory is similar to the classical trajectory given by an inversed pendulum model. On the $z$ axis, the trajectory is at the height of the CoM; however, it is is lowered when the reference foot is lifted above the ground, to keep the same CoM height.

In Fig. 11, the evolution of the ZMP position and the CoM position are plotted with respect to footprints of the humanoid robot. The obtained walking motion is stable and robust. The robot produces the desired walking pattern. It is worth to note that the robot falls down for the same scenario if the stabilizer was not active.

\section{CONCLUSION AND FUTURE WORK}

This paper deals with a new efficient stabilizer for humanoid robots. The proposed solution is based on a nonlinear PID based regulation, coupled with a spherical projection of the ZMP regulation error in the CoM jacobian control.

The main advantage of this stabilizer lies in the enhancement of the stability of the robot under the control of a previously proposed control framework for wholebody motions control [25]. The obtained results are very promising. 


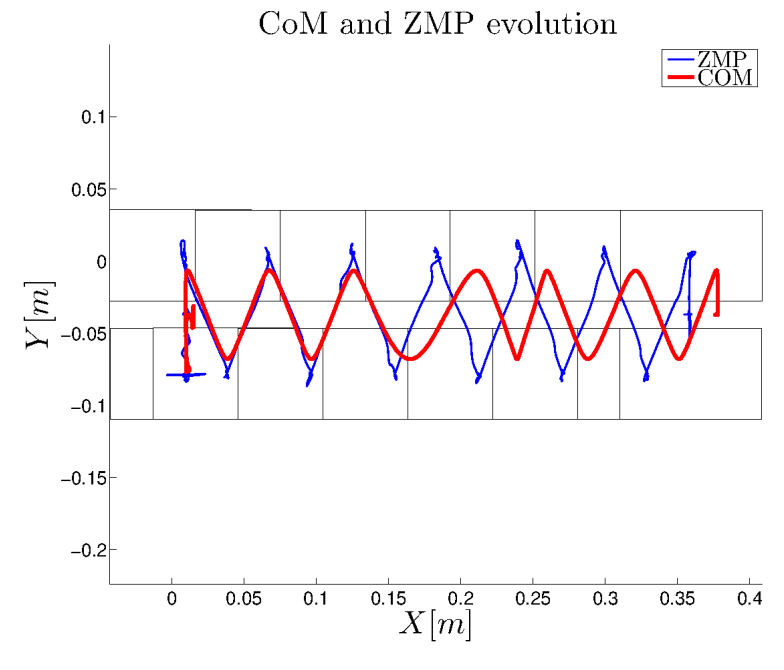

Fig. 11: Evolution of ZMP and CoM trajectories with footprint displayed (scenario 2).

In future work, we aim at extending this work for more complex scenarios including carrying objects or in humanrobot interactions.

\section{REFERENCES}

[1] S. Ma, T. Tomiyama, and H. Wada, "Omnidirectional static walking of a quadruped robot," IEEE Transactions on Robotics, vol. 21, no. 2, pp. 152-161, 2005.

[2] M. Vukobratovíc, B. Borovac, and D. Surla, Biped locomotion: dynamics, stability, control, and application, ser. Scientific Fundamentals of Robotics. Springer-Verlag, 1990.

[3] M. Vukobratovic and B. Borovac, "Zero-moment point-thirty five years of its life," International Journal of Humanoid Robotics, vol. 1, no. 1, pp. 157-173, 2004.

[4] P. Sardain and G. Bessonnet, "Forces acting on a biped robot. Center of pressure-zero moment point," IEEE Transactions on Systems, Man and Cybernetics, Part A: Systems and Humans, vol. 34, no. 5, pp. 630-637, 2004.

[5] S. Kajita, O. Matsumoto, and M. Saigo, "Real-time 3D walking pattern generation for a biped robot with telescopic legs," in Proceedings of the IEEE International Conference on Robotics and Automation (ICRA'01), vol. 3. IEEE, 2001, pp. 2299-2306.

[6] Z. Tang and M. Er, "Humanoid 3D Gait Generation Based on Inverted Pendulum Model," in Proceedings of the IEEE 22nd International Symposium on Intelligent Control (ISIC'07), Singapore, 2007, pp. 339-344.

[7] S. Kajita, F. Kanehiro, K. Kaneko, K. Yokoi, and H. Hirukawa, "The 3D Linear Inverted Pendulum Mode: A simple modeling for a biped walking pattern generation," in Proceedings of the IEEE/RSJ International Conference on Intelligent Robots and Systems (IROS'01), Maui, Hawaii, USA, 2001, pp. 239-246.

[8] S. Feng and Z. Sun, "A simple trajectory generation method for biped walking," in Proceedings of the 10th International Conference on Control, Automation, Robotics and Vision (ICARCV'08), Hanoi, Vietnam, 2008, pp. 2078-2082.

[9] S. Kajita, F. Kanehiro, K. Kaneko, K. Fujiwara, K. Yokoi, and H. Hirukawa, "Biped walking pattern generation by a simple threedimensional inverted pendulum model," Advanced Robotics, vol. 17, no. 2, pp. 131-147, 2003.

[10] D. T. Butterworth, B. S. Han, and S. P. Singh, "Predictably unpredictable? on the implementation of a walking pattern generator for the full-sized humanoid robot hubo2 using model predictive control."

[11] Z. Li, B. Vanderborght, N. G. Tsagarakis, L. Colasanto, and D. G. Caldwell, "Stabilization for the compliant humanoid robot COMAN exploiting intrinsic and controlled compliance," in Proceedings of the IEEE International Conference on Robotics and Automation (ICRA'12). IEEE, 2012, pp. 2000-2006.
[12] Z. Li, N. G. Tsagarakis, and D. G. Caldwell, "A passivity based admittance control for stabilizing the compliant humanoid COMAN," in Proceedings of the IEEE-RAS International Conference on Humanoid Robots (Humanoids'12). IEEE, 2012, pp. 43-49.

[13] S. Hyon, "Compliant terrain adaptation for biped humanoids without measuring ground surface and contact forces," IEEE Transactions on Robotics, vol. 25, no. 1, pp. 171-178, 2009.

[14] Q. Huang and Y. Nakamura, "Sensory reflex control for humanoid walking," IEEE Transactions on Robotics, vol. 21, no. 5, pp. 977984, 2005.

[15] Y.-D. Kim, B.-J. Lee, J.-K. Yoo, J.-H. Kim, and J.-H. Ryu, "Compensation for the landing impact force of a humanoid robot by time domain passivity approach," in Proceedings of the IEEE International Conference on Robotics and Automation (ICRA'06). IEEE, 2006, pp. $1225-1230$.

[16] K. Hirai, M. Hirose, Y. Haikawa, and T. Takenaka, "The development of honda humanoid robot," in Proceedings of the IEEE International Conference on Robotics and Automation (ICRA'98), vol. 2. IEEE, 1998, pp. 1321-1326.

[17] T. Buschmann, S. Lohmeier, and H. Ulbrich, "Humanoid robot lola: Design and walking control," Journal of Physiology-Paris, vol. 103, no. 3, pp. 141-148, 2009.

[18] B.-K. Cho, J.-H. Kim, and J.-H. Oh, "Online balance controllers for a hopping and running humanoid robot," Advanced Robotics, vol. 25, no. 9-10, pp. 1209-1225, 2011.

[19] S.-H. Lee and A. Goswami, "Ground reaction force control at each foot: A momentum-based humanoid balance controller for non-level and non-stationary ground," in Proceedings of the IEEE/RSJ International Conference on Intelligent Robots and Systems (IROS'10). IEEE, 2010, pp. 3157-3162.

[20] B. Stephens, "Push recovery control for force-controlled humanoid robots," Ph.D. dissertation, Carnegie Mellon University, 2011.

[21] Y. Choi, B.-J. You, and S.-R. Oh, "On the stability of indirect ZMP controller for biped robot systems," in Proceedings of the IEEE/RSJ International Conference on Intelligent Robots and Systems (IROS'04), vol. 2. IEEE, 2004, pp. 1966-1971.

[22] Y. Choi, D. Kim, and B.-J. You, "On the walking control for humanoid robot based on the kinematic resolution of com jacobian with embedded motion," in Proceedings of the IEEE International Conference on Robotics and Automation (ICRA'06). IEEE, 2006, pp. 2655-2660.

[23] B.-J. Lee, D. Stonier, Y.-D. Kim, J.-K. Yoo, and J.-H. Kim, "Modifiable walking pattern of a humanoid robot by using allowable ZMP variation," IEEE Transactions on Robotics, vol. 24, no. 4, pp. 917-925, 2008.

[24] S.-J. Yi, B.-T. Zhang, D. Hong, and D. D. Lee, "Learning full body push recovery control for small humanoid robots," in Proceedings of the IEEE International Conference on Robotics and Automation (ICRA'11). IEEE, 2011, pp. 2047-2052.

[25] D. Galdeano, A. Chemori, S. Krut, and P. Fraisse, "Task-based wholebody control of humanoid robots with ZMP regulation, real-time application to a squat-like motion," in 11th edition of the International Conference on Systems, Analysis and Automatic Control (SSD SAC 2014), Castelldefels-Barcelona - Spain, 2014.

[26] Y. Nakamura, H. Hanafusa, and T. Yoshikawa, "Task-priority based redundancy control of robot manipulators," The International Journal of Robotics Research, vol. 6, no. 2, pp. 3-15, 1987.

[27] N. Mansard, O. Stasse, P. Evrard, and A. Kheddar, "A versatile generalized inverted kinematics implementation for collaborative working humanoid robots: The stack of tasks," in Proceedings of IEEE International Conference on Advanced Robotics (ICAR'09)., 2009, pp. $1-6$.

[28] Y. Xu, J. Hollerbach, and D. Ma, "A nonlinear PD controller for force and contact transient control," Control Systems, IEEE, vol. 15, no. 1, pp. 15-21, 1995.

[29] H. Seraji, "A new class of nonlinear PID controllers with robotic applications," Journal of Robotic Systems, vol. 15, no. 3, pp. 161181, 1998.

[30] Y. Su, D. Sun, and B. Duan, "Design of an enhanced nonlinear PID controller," Mechatronics, vol. 15, no. 8, pp. 1005-1024, 2005.

[31] W. Shang and S. Cong, "Nonlinear computed torque control for a high-speed planar parallel manipulator," Mechatronics, vol. 19, no. 6, pp. 987-992, 2009. 